\title{
ASMq protects against early burn wound progression in rats by alleviating oxidative stress and secondary mitochondria-associated apoptosis via the Erk/p90RSK/Bad pathway
}

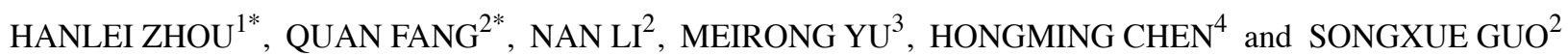 \\ Departments of ${ }^{1}$ Vascular Surgery and ${ }^{2}$ Plastic Surgery, The Second Affiliated Hospital Zhejiang University \\ School of Medicine; ${ }^{3}$ Clinical Research Center, The Second Affiliated Hospital Zhejiang University School of Medicine; \\ ${ }^{4}$ Department of Plastic Surgery, International Medical Center of The Second Affiliated Hospital Zhejiang University \\ School of Medicine, Hangzhou, Zhejiang 310009, P.R. China
}

Received August 7, 2020; Accepted February 23, 2021

DOI: $10.3892 / \mathrm{mmr} .2021 .12029$

\begin{abstract}
Burn wounds present an evolutionary progression, in which the initial wound tissue deepens and expands following thermal injury. Progressive tissue damage in the zone of stasis may worsen burn injury, which is associated with oxidative stress and secondary apoptosis, and worsen the prognosis of patients with burn wounds. The mitochondrial apoptotic pathway is involved in receiving oxidative signals and regulating tissue apoptosis. Previously, Abnormal Savda Munziq (ASMq), a natural compound of traditional Uyghur Medicine, which includes ten types of herb, has been reported to exhibit a number of effects, including anti-inflammatory, antioxidative and anti-apoptotic activities. The present study demonstrated that ASMq protected against early burn wound progression following thermal injury in rats; this effect may be mediated by its ability to attenuate oxidative stress-induced mitochondria-associated apoptosis. The present study may provide a novel therapeutic method to prevent early burn wound progression following burn injury.
\end{abstract}

\section{Introduction}

Burn wound progression, which was first introduced by DM Jackson in 1953, refers to the deterioration of tissue damage in the zone of stasis, especially during the first few days following thermal injury (1). According to Jackson's model of

Correspondence to: Dr Songxue Guo, Department of Plastic Surgery, The Second Affiliated Hospital Zhejiang University School of Medicine, 88 Jiefang Road, Hangzhou, Zhejiang 310009, P.R. China

E-mail:sxguo@zju.edu.cn

${ }^{*}$ Contributed equally

Key words: burn-wound progression, oxidative stress, apoptosis, abnormal savda munziq, Erk pathway, rats thermal wound injury, the burn wound is divided into three concentric zones: A central zone of coagulation, an intermediate zone of stasis and an outer zone of hyperemia. The central zone is damaged by thermal injury directly and suffers irreversible coagulation necrosis with thrombosed vessels; the zone of hyperemia is characterized by spontaneous recovery, while the zone of stasis is considered salvageable via optimal intervention (2). The recovery of the zone of stasis is vital in preventing irreversible damage and determining the final severity of the wound. Burn wound progression contributes to the development of wound contraction, hypertrophic scarring, wound infection and sepsis. Therefore, appropriate and timely interventions to prevent burn wound progression are of clinical significance for treatment of the wound and prognosis of patients with burns (3), as well as for the early prevention of hypertrophic scar formation due to burn injury (4). Multiple mechanisms are involved in burn wound progression, including oxygen free radical-induced damage, hypoperfusion, micro-thrombosis and vasoconstriction, which can lead to early inflammatory cascades and cell death (apoptosis or necrosis) $(3,5,6)$. Mitochondria are important organelles in eukaryotic cells that are susceptible to oxidative stress by regulating energy metabolism and apoptosis (7). Our previous studies demonstrated the effectiveness of protective strategies against oxidative damage and tissue apoptosis in preventing burn wound conversion $(8,9)$.

Abnormal Savda Munziq (ASMq), a traditional herbal formula in Uyghur medicine, primarily consists of ten herbs, including Lavandula angustifolia, Foeniculum vulgare, Anchusa italica, Euphorbia humifusae, Melissa officinalis, Adiantum capillus-veneris, Glycyrrhiza uralensis, Cordia dichotoma, Ziziphus jujuba and Alhagi pseudoalhagi (10). Previous reports demonstrated that ASMq exerts antioxidative, anti-inflammatory and anti-apoptotic properties to inhibit the formation of hypertrophic scars (4) and treat diabetes, cardiovascular disease, pulmonary disorders and various types of cancer, such as cervical cancer and hepatic carcinoma (10-17); to the best of our knowledge, however, few studies have investigated its potential effects on burn injury. In consideration of the critical role of reactive oxygen species (ROS)-induced 
oxidative stress and subsequent mitochondria-associated apoptosis in the pathophysiological development of early burn wounds, it was hypothesized that ASMq may prevent early burn wound progression in rats by suppressing oxidative stress and apoptosis. The present study aimed to evaluate the possible mechanisms of action and regulation.

The present study aimed to demonstrate that ASMq may be an effective drug to ameliorate early burn wound progression, thus avoiding or alleviating adverse outcomes associated with burn wounds.

\section{Materials and methods}

Experimental animals. Adult male Sprague-Dawley (SD) rats weighing 200-220 g were used according to an experimental protocol approved by The Second Affiliated Hospital, Zhejiang University, School of Medicine, Institutional Animal Care and Use Committee (approval no. 2018-095) in accordance with the National Institutes of Health Guidelines for the Care and Use of Laboratory Animals (18). The animals were housed in a 12-h light/dark cycle in a filtered-air unit with consistent temperature $\left(20-26^{\circ} \mathrm{C}\right)$ and humidity (50-65\%) and ad libitum access to food and water. All animals were purchased from Shanghai SLAC Laboratory Animal Co., Ltd.

'Comb' burn model establishment, animal grouping and drug administration. To create a row of four bands of full-thickness thermal burn injuries $(20 \times 10 \mathrm{~mm})$ with three interspaces of uninjured skin $(20 \times 5 \mathrm{~mm})$, a customized rectangular brass comb was boiled in $100^{\circ} \mathrm{C}$ water for $5 \mathrm{~min}$, and then applied to the shaved dorsum skin of rats for $10 \mathrm{sec}$ following anesthesia with intraperitoneal injection of $1 \%$ pentobarbital sodium at a dose of $30 \mathrm{mg} / \mathrm{kg}$, as previously described (19-22). The breathing and heart rates of experimental rats were monitored during anesthesia. The rats received immediate postoperative analgesia with buprenorphine $(0.03 \mathrm{mg} / \mathrm{kg}$, subcutaneous), followed by continuous analgesia with buprenorphine $(0.03 \mathrm{mg} / \mathrm{kg}$, subcutaneous) every $12 \mathrm{~h}$. The interstices of undamaged skin represented the zone of stasis. Animals in the sham group were treated with exposure to $25^{\circ} \mathrm{C}$ water following anesthesia.

A total of $64 \mathrm{SD}$ rats were randomly assigned to the following eight groups ( $n=8$ per group): i) Sham; ii) burn; iii) sham + AMSq; iv) burn + low dosage AMSq (400 mg/kg); v) burn + medium dosage AMSq (800 mg/kg); vi) burn + high dosage AMSq (1,600 mg/kg); vii) burn + AMSq + lipopolysaccharide (LPS) and viii) burn + AMSq + PD98059.

No specific drugs were administered following hot water exposure in the burn group. ASMq powder (purchased from Xinjiang Uygur Pharmaceutical Co., Ltd.; State Food and Drug Administration approval no. Z65020166) was dissolved in double-distilled water to create a $100 \mathrm{mg} / \mathrm{ml}$ suspension.

The feed suspension was prepared $1 \mathrm{~h}$ before the operation according to the weight of the rats and ASMq dosage. The volume of suspension was set as $2 \mathrm{ml}$ and the ASMq was diluted using double-distilled water. The rats in different burn groups with various doses of ASMq were fed $2 \mathrm{ml} \mathrm{ASMq}$ suspension at a dosage of 400,800 or $1,600 \mathrm{mg} / \mathrm{kg}$. The selection of ASMq dose was based on a previous study (23). For the burn + ASMq + LPS group, LPS (Sigma-Aldrich;
Merck $\mathrm{KGaA}$ ) was dissolved in $0.9 \%$ normal saline and administered to rats via intraperitoneal injection at a dose of $10 \mathrm{mg} / \mathrm{kg}$ following the operation. PD98059, a specific inhibitor of extracellular-regulated protein kinase (Erk) (24), was used to identify potential mechanisms of apoptosis in the zone of stasis during burn wound progression. In addition to ASMq suspension administration, the rats in burn + AMSq + PD98059 group also received a caudal intravenous injection of PD98059 (Sigma-Aldrich; Merck KGaA) dissolved in dimethylsulfoxide at a dose of $0.3 \mathrm{mg} / \mathrm{kg}$ (once/day for 3 days before the operation). All animals were sacrificed at $24 \mathrm{~h}$ by overdose of sodium pentobarbital (200 mg/kg, intraperitoneal), according to a previous study (25).

Tissue collection and preparation. Skin tissue specimens from wounds were harvested $48 \mathrm{~h}$ after operation, according to our previous study $(8,9)$. One band of the interspace skin with $2 \mathrm{~mm}$ burned tissue on each side $(10 \times 9 \mathrm{~mm})$ was collected from each burn model and stored in $10 \%$ formalin at $4^{\circ} \mathrm{C}$ for subsequent immunohistochemistry analysis, while the remaining two bands of interspace skin (without burn tissue; 10x5 mm) from each burned rat were stored at $-80^{\circ} \mathrm{C}$ for molecular biological assessments. A total of three corresponding areas of unburnt skin from each rat of the sham group were used as controls.

Histological assessment. Rat skin tissue was prepared by fixation in $4 \%$ formaldehyde solution for $12 \mathrm{~h}$ at $4{ }^{\circ} \mathrm{C}$. The tissue samples were then dehydrated in a graded series of ethanol solutions, embedded in paraffin and cut into $3-\mu \mathrm{m}$ sections. The hematoxylin and eosin (H\&E) staining was performed according to routine procedure. Histological changes were evaluated under a light microscope (DM2500; Leica Microsystems $\mathrm{GmbH}$ ). A total of five fields (magnification, $\mathrm{x} 200$ ) were selected randomly for observation from each slice.

Oxidative stress evaluation. The frozen tissue samples from rats were crushed, ground and homogenized for 8 min using a tissue homogenizer with homogenization buffer $\left(4^{\circ} \mathrm{C}\right)$, which included $1 \mathrm{mmol} / 1$ phenylmethylsulfonyl fluoride, $1 \mathrm{mg} / \mathrm{ml}$ pepstatin $\mathrm{A}, 1 \mathrm{mg} / \mathrm{ml}$ aprotinin and $1 \mathrm{mg} / \mathrm{ml}$ leupeptin in phosphate-buffered saline (PBS; $\mathrm{pH}, 7.2$ ) containing $0.5 \%$ sodium deoxycholate and $1 \%$ Triton $\mathrm{X}-100$, at a ratio of $100 \mathrm{mg}$ tissue $/ \mathrm{ml}$. Following incubation at $4^{\circ} \mathrm{C}$ for $1 \mathrm{~h}$, the final homogenate was centrifuged at $10,000 \mathrm{x}$ g for $15 \mathrm{~min}$ at $4^{\circ} \mathrm{C}$ and the supernatants were used for detection kit assays. The total protein concentration was determined using a BCA assay kit (Nanjing KeyGen Biotech Co., Ltd.).

In order to evaluate lipid peroxidation of skin tissue, malondialdehyde (MDA) levels were measured. Skin tissue homogenates from the burn wounds were incubated with a thiobarbituric acid reactive species assay kit reagent (cat. no. KGT003-1; Nanjing KeyGen Biotech Co., Ltd.) to determine MDA levels, which were expressed in $\mathrm{nmol} / \mathrm{mg}$ protein. Levels of tissue superoxide dismutase (SOD) and glutathione peroxidase (GPx) were evaluated to determine the oxidative stress status in the skin tissues of burn wounds and were detected using commercial assay kits from Nanjing KeyGen Biotech Co., Ltd. (cat. nos. KGT00150 and KGT-014, respectively) according to the manufacturer's protocols. The results 
were expressed in $\mathrm{U} / \mathrm{mg}$ for SOD and $\mathrm{nmol} / \mathrm{min} / \mathrm{mg}$ for GPx. Absorbance values were measured using a microplate reader (MDA, 532 nm; SOD, 550 nm; GPx, 412 nm) (Model 680 Microplate Reader; Bio-Rad Laboratories, Inc.).

Immunofluorescence staining. In order to determine the location and quantity of the specific antigens in the interspace skin following burn injury, the prepared slices were washed in PBS for $10 \mathrm{~min}$, followed by boiling at $95^{\circ} \mathrm{C}$ in $0.01 \mathrm{mmol}$ citrate buffer $(\mathrm{pH}, 6.0)$ for $10 \mathrm{~min}$ for antigen retrieval. Then, the slices was incubated with hydrogen peroxide for $10 \mathrm{~min}$ at room temperature, and 5\% bovine serum albumin (Gibco; Thermo Fisher Scientific, Inc.) was applied later as the blocking solution for $20 \mathrm{~min}$ at room temperature. Without being washed, the sections were incubated with anti-phosphorylated (p)-Erk (1:200; cat. no. 4370; Cell Signaling Technology, Inc.) or anti-p-Bad (1:200; cat. no. sc-12969-R; Santa Cruz Biotechnology, Inc.) antibodies overnight at $4^{\circ} \mathrm{C}$. After being rinsed with PBS, the sections were incubated with FITC-(1:50; cat. no. BA1105; Wuhan Boster Biological Technology, Ltd.) or Cy3-(1:50; cat. no. BA1032; Wuhan Boster Biological Technology, Ltd.) labeled goat anti-rabbit secondary antibodies for $2 \mathrm{~h}$ at $37^{\circ} \mathrm{C}$ in the dark. The sections were rinsed and stained with DAPI (100 ng/ml; Wuhan Boster Biological Technology, Ltd.) for $8 \mathrm{~min}$ at room temperature, and then mounted with VECTASHIELD ${ }^{\circledR}$ mounting medium (Wuhan Boster Biological Technology, Ltd.). All slices were observed and photographed under a fluorescence microscope (magnification, x200) (DM5500B; Leica Microsystems $\mathrm{GmbH}$ ).

Terminal deoxynucleotidyl-transferase-mediated dUTP nick end labeling (TUNEL) staining. TUNEL staining was performed using a commercial cell death detection kit (Roche Diagnostics) according to the manufacturer's protocols. Briefly, after deparaffinization and rehydration, the slices were pretreated by proteinase K (PCR grade; Roche Diagnostics) for $15 \mathrm{~min}$ at room temperature. Then, the permeabilization was conducted with fresh prepared $0.1 \%$ Triton-X-100 and $0.1 \%$ sodium citrate for $8 \mathrm{~min}$ at room temperature. Then, incubation with trypsin $(0.01 \mathrm{~N} \mathrm{HCL})$ was applied for $30 \mathrm{~min}$ at $37^{\circ} \mathrm{C}$. Subsequently, the slices were transferred into a container with $0.1 \mathrm{M}$ citrate buffer $(\mathrm{pH}, 6)$ and received microwave irradiation $(350 \mathrm{~W})$ for $5 \mathrm{~min}$. Rinsed slices were treated with TUNEL mixture for $60 \mathrm{~min}$ at $37^{\circ} \mathrm{C}$ in a humidified atmosphere in the dark. Finally, the signal conversion was handled with the help of converter-POD and DAB substrate. The stained slices were observed by a light microscope (magnification, x400; DM2500; Leica Microsystems GmbH) and images were captured. The index of apoptosis was calculated as the percentage of apoptotic cells among all cells counted. Cells with dark brown-stained nuclei were counted by two independent investigators who were blinded to the group assignments. At least three visual fields per slide and five slides per group were evaluated by the investigators.

Western blot analysis. Frozen skin samples were harvested from rats for western blotting. Briefly, samples were cut into pieces $(\sim 1 \mathrm{x} 1 \mathrm{~mm})$ and lysed with RIPA lysis buffer (cat. no. AR0105; Wuhan Boster Biological Technology, Ltd.) for $1 \mathrm{~h}$ on ice, then the lysates were centrifuged at $14,000 \mathrm{x}$ g for $10 \mathrm{~min}$ at $4^{\circ} \mathrm{C}$. The protein samples $(50 \mu \mathrm{g})$ were mixed with loading buffer and subjected to SDS-PAGE on 6-12\% gels, and subsequently transferred onto nitrocellulose membranes by electrophoresis, while aliquots of samples were used to determine the protein concentration of each sample using a bicinchoninic acid kit (cat. no. KGPBCA; Nanjing KeyGen Biotech Co., Ltd.). Subsequently, membranes were incubated in blocking buffer (5\% skimmed milk) for $2 \mathrm{~h}$ at room temperature, and incubated overnight at $4^{\circ} \mathrm{C}$ with the following primary antibodies: Anti-XO (1:5,000; cat. no. ab109235; Abcam), anti-Nox4 (1:2,000; cat. no. ab109225; Abcam), anti-Bad (1:1,000; cat. no. sc-8044; Santa Cruz Biotechnology, Inc.), anti-p-Bad (1:1,000; cat. no. sc-12969-R; Santa Cruz Biotechnology, Inc.), anti-cytochrome $c$ (Cyto C; 1:1,000; cat. no, sc-7159; Santa Cruz Biotechnology, Inc.), anti-cleaved caspase (CC)3 (1:1,000; cat. no. 9664; Cell Signaling Technology, Inc.), anti-CC9 (1:1,000; cat. no. 9509; Cell Signaling Technology, Inc.), anti-Erk1/2 (1:1,000; cat. no. 9102; Cell Signaling Technology, Inc.), anti-p-Erk1/2 (1:1,000; cat. no. 9101; Cell Signaling Technology, Inc.) and anti-p90 ribosomal S6 kinase (p90RSK; 1:1,000; cat. no. ab32114; Abcam). $\beta$-actin (1:2,000; cat. no. SC-47778; Santa Cruz Biotechnology, Inc.) was used as a control. Following incubation with a HRP-conjugated secondary antibody (1:5,000; cat. no. 31160; Pierce; Thermo Fisher Scientific, Inc.) for $1 \mathrm{~h}$ at room temperature, the bands were detected with West Dura Extended Duration substrate (Pierce; Thermo Fisher Scientific, Inc.) and X-ray film (Kodak), then analyzed by Bandscan 5.0 software (Agilent Technologies, Inc.) for comparison against $\beta$-actin.

Statistical analysis. GraphPad Prism version 7 software (GraphPad Software, Inc.) was used for statistical analysis. Data are presented as the mean \pm SD. Multiple comparisons among the sham and burn groups were analyzed with one-way ANOVA followed by Bonferroni's post hoc test. $\mathrm{P}<0.05$ was considered to indicate a statistically significant difference.

\section{Results}

ASMq alleviates tissue damage of skin in the zone of stasis of rat burn wounds. H\&E staining demonstrated thinning of the epidermis layer, swelling of the dermis layer, alteration of collagen structure, infiltration of inflammatory cells and tissue destruction in the interspaces between the two wounds following thermal injury. ASMq alleviated these changes in a dose-dependent manner (Fig. 1).

Effect of ASMq on early elevated oxidative stress in the zone of stasis of rat burn wounds. MDA, an indicator of lipid peroxidation, indicates the severity of oxidative damage (26). There was no significant difference in the levels of MDA between the sham + high dosage AMSq $(1,600 \mathrm{mg} / \mathrm{kg})$ and sham groups. Although low dosage AMSq $(400 \mathrm{mg} / \mathrm{kg}) \mathrm{did}$ not significantly alleviate the increase of MDA levels, high and medium dosage ASMq significantly decreased the levels of MDA in a dose-dependent manner ( $\mathrm{P}<0.01$; Fig. 2A).

Low dosage AMSq $(400 \mathrm{mg} / \mathrm{kg})$ did not influence the activity levels of endogenous antioxidant enzymes (GPX and SOD) in 


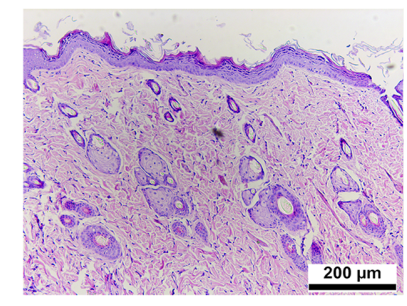

Sham

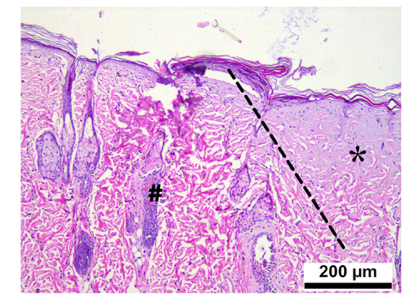

ASMq low

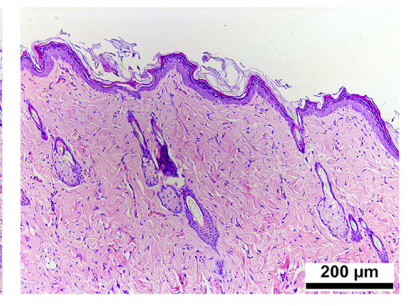

Sham+ASMq

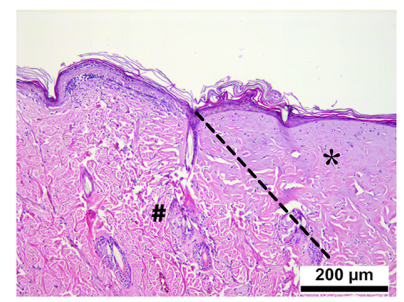

ASMq medium

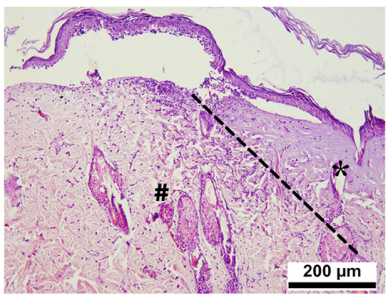

Burn $48 \mathrm{~h}$

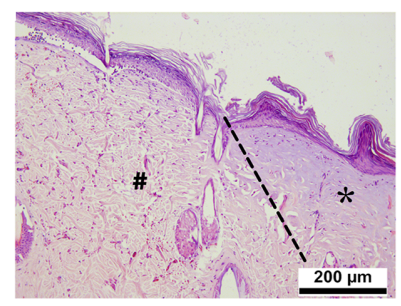

ASMq high

Figure 1. Histological assessment of burn skin samples in the early stages following severe burn and ASMq application. Representative hematoxylin and eosin staining images show the typical histological changes in the different groups ( $\mathrm{n}=8 /$ group). The progression boundaries are indicated by dotted lines, the stasis zones are marked with \# and the initial burn regions are marked with *, presenting notable hyalinization. Scale bar, $200 \mu \mathrm{m}$. ASMq, Abnormal Savda Munziq.

A

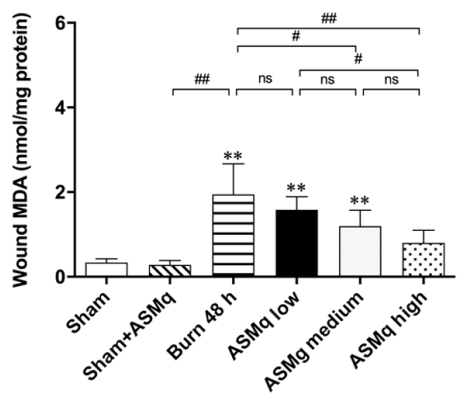

D

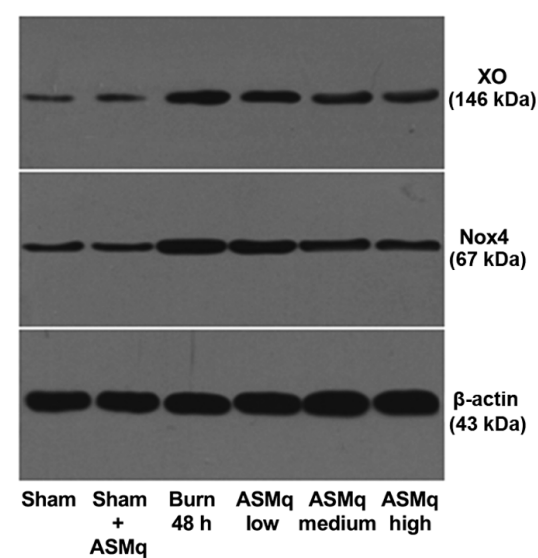

B
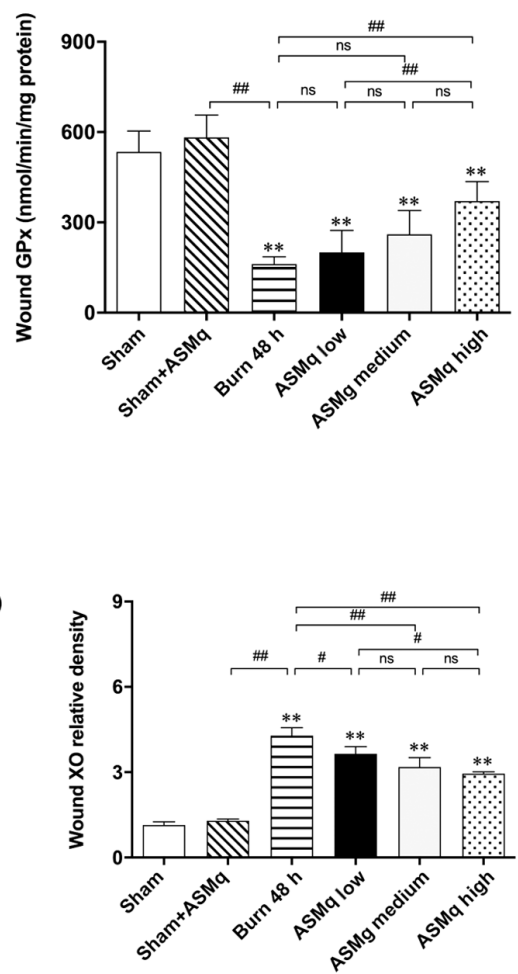

C
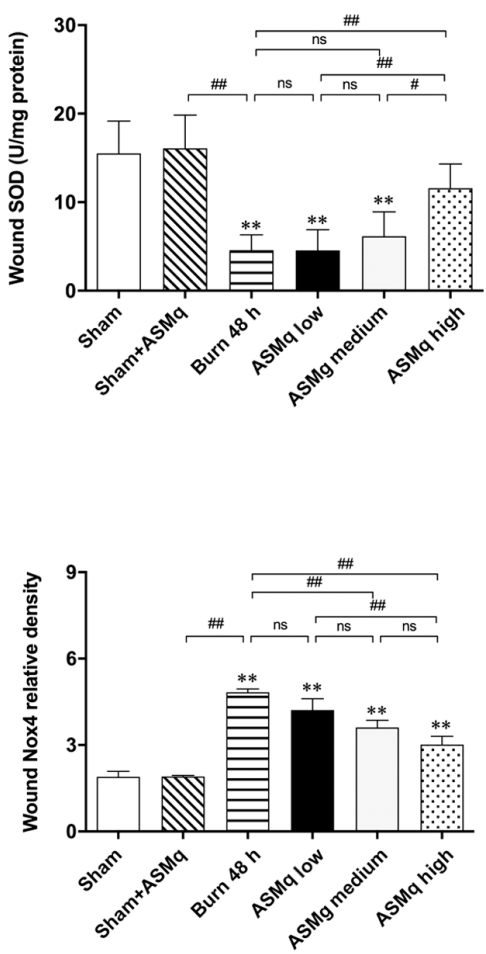

Figure 2. Assessment of oxidative stress within the zone of stasis and the effects of ASMq. (A-C) ASMq downregulates MDA levels and upregulates the activity of endogenous antioxidant enzymes (GPx, SOD) in wounds and (D) downregulates the expression of XO and Nox4 in a dose-dependent manner following burn injury. Data are presented as the mean $\pm \mathrm{SD}$ ( $\mathrm{n}=8 /$ group). ${ }^{* *} \mathrm{P}<0.01$ vs. sham; ${ }^{\text {P }}<0.05$, ${ }^{\#} \mathrm{P}<0.01$. ASMq, Abnormal Savda Munziq; XO, xanthine oxidase; Nox, NADPH oxidase; ns, not significant; MDA, malondialdehyde; SOD, superoxide dismutase; GPx, glutathione peroxidase.

the healthy skin of rats. High dosage AMSq $(1,600 \mathrm{mg} / \mathrm{kg})$ upregulated the activity of endogenous antioxidant enzymes (GPX and SOD) in the zone of stasis of rat burn wounds, while no significant differences were detected in medium and lower dose groups (Fig. 2B and C).
$\mathrm{XO}$, which is associated with the generation of ROS (27), was semi-quantified by western blotting (Fig. 2D). The results demonstrated that XO expression levels in the zone of stasis of rat burn wounds were significantly downregulated by ASMq. There was no significant difference in the expression levels 
B

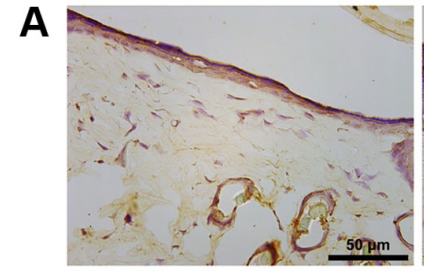

Sham

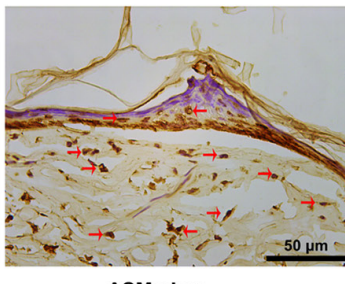

ASMq low

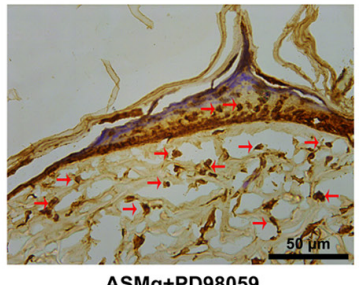

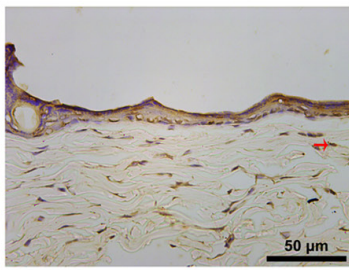

Sham+ASMq

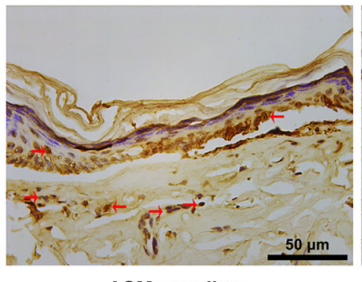

ASMq medium

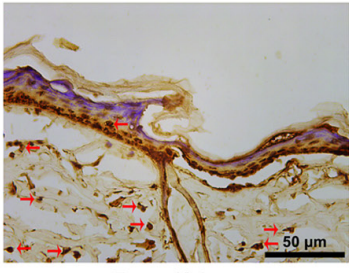

Burn $48 \mathrm{~h}$

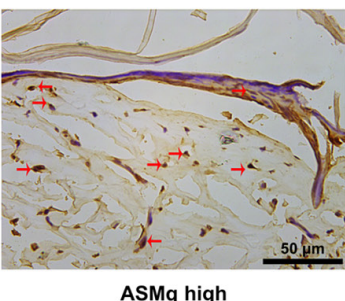

ASMq high

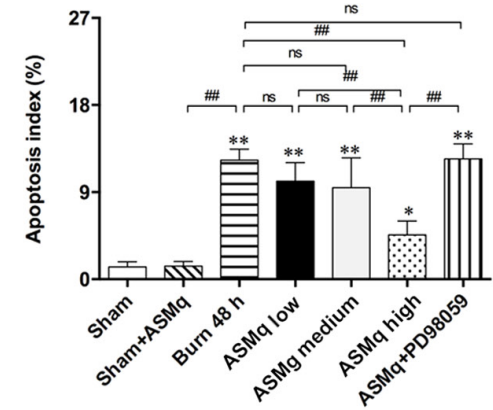

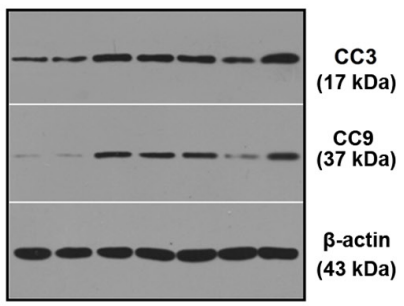
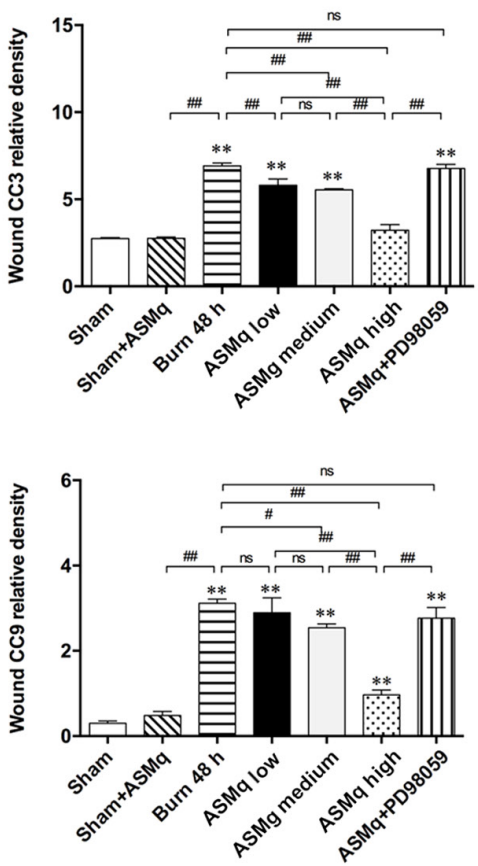

Figure 3. Evaluation of apoptosis within the zone of stasis in different groups. (A) Representative images of TUNEL-stained slices and ratio of apoptotic cells. Red arrows indicate stained nuclei of apoptotic cells. (B) Western blot results for CC3 and CC9 post-burn. Scale bar, $50 \mu \mathrm{m}$. Data are presented as the mean $\pm \mathrm{SD}$ ( $\mathrm{n}=8$ /group). ${ }^{*} \mathrm{P}<0.05,{ }^{* *} \mathrm{P}<0.01$ vs. sham; ${ }^{\#} \mathrm{P}<0.05,{ }^{\# \#} \mathrm{P}<0.01$. CC, cleaved caspase; ASMq, Abnormal Savda Munziq; ns, not significant.

of XO between medium and low dosage or medium and high dosage ASMq $(\mathrm{P}>0.05)$. XO levels in the high dosage ASMq group were lower than those in the low dosage ASMq group $(\mathrm{P}<0.05)$. The effect of decreasing XO levels induced by burn injury was more significant in the high dosage ASMq group $(\mathrm{P}<0.01)$. Nox 4 is an important member of the Nox family that can be used to indicate mitochondrial status (28). The effect of ASMq on Nox4 expression was similar to that of XO: Specifically, both high and medium dosage ASMq significantly decreased expression levels of Nox4 in the zone of stasis (Fig. 2D).

ASMq dose-dependently decreases cell apoptosis in burn wound tissue of rats. TUNEL staining demonstrated that the number of apoptotic cells with brown-stained nuclei in burn wounds increased. According to the random multi-field cell count, high dosage ASMq significantly decreased the number of apoptotic cells in the zone of stasis of skin tissue compared with the number observed in the burn group, while statistically insignificant differences were observed in the medium and low dosage groups (Fig. 3A).

Western blot analysis was performed to determine the protein expression levels of CC3 and CC9, which are key signaling molecules in the execution phase of mitochondria-associated cell apoptosis (29). Burn injury significantly increased CC3 and CC9 expression levels. Increasing doses of ASMq resulted in decreased CC3 expression levels, with a peak observed in the group treated with a dose of $1,600 \mathrm{mg} / \mathrm{kg}$. The expression levels of CC 9 were significantly decreased by high and medium dosage ASMq (Fig. 3B).

Pretreatment with PD98059 partially reversed the mitigative effect of high dosage ASMq on cell apoptosis and expression levels of $\mathrm{CC} 3$ and $\mathrm{CC} 9$ in the zone of stasis of skin tissue following burn injury. PD98059 reversed the decrease in pro-apoptotic protein (Cyto $\mathrm{C}$ ) in the burn wound induced by high dosage ASMq (Fig. 3A). These results suggested a potential involvement of Erk signaling in regulating tissue apoptosis in the zone of stasis.

ASMq dose-dependently increases the ratio of $p$-Bad/Bad and release of mitochondrial Cyto $C$. The aforementioned results indicated that the anti-apoptotic effect of ASMq may be associated with the regulation of mitochondria-associated apoptosis pathways involved in Bad. Western blotting results showed that ASMq altered the Bad/Cyto C signaling molecules cascade. The ratio of $\mathrm{p}$-Bad to Bad increased significantly in skin tissue following burn injury. All three dosages of ASMq increased the ratio of $\mathrm{p}-\mathrm{Bad} / \mathrm{Bad}$ in a dose-dependent manner. Secondly, a significant increase in expression levels of Cyto $\mathrm{C}$ was observed following burn injury, which was alleviated by medium and high dosage ASMq in a dose-dependent manner. PD98059 antagonized the effect of high-dosage ASMq (Fig. 4). 

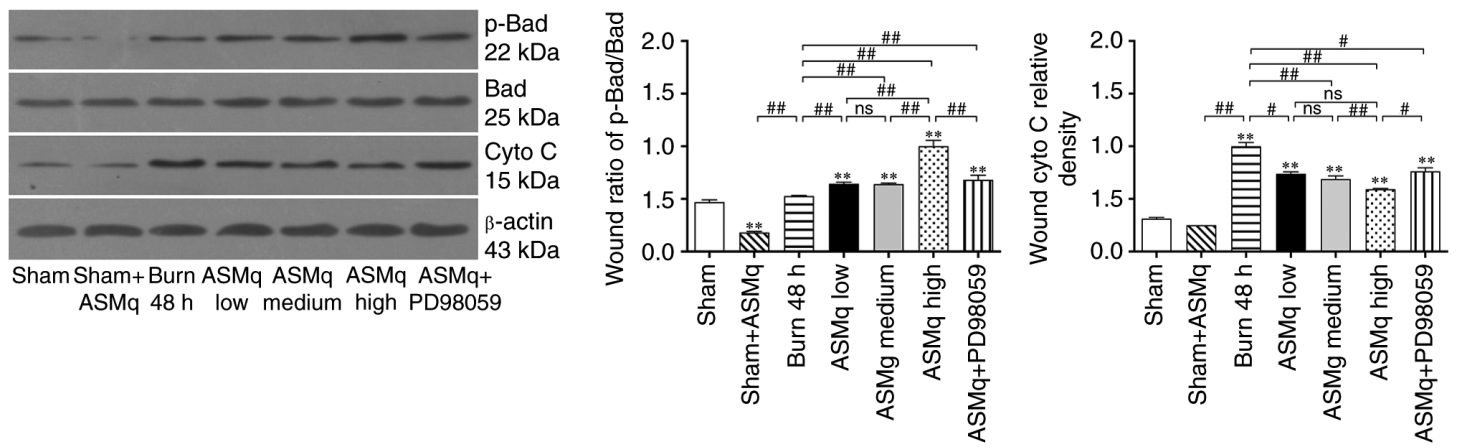

Figure 4. ASMq increases the ratio of p-Bad/Bad and release of mitochondrial Cyto C, whereas PD98059 reverses the effect of high-dosage ASMq. Western blot bands and relative $\mathrm{p}-\mathrm{Bad} / \mathrm{Bad}$ ratios in different groups. Data are presented as the mean $\pm \mathrm{SD}$ (n=8/group). ${ }^{* *} \mathrm{P}<0.01$ vs. sham; ${ }^{\#} \mathrm{P}<0.05$, ${ }^{\# \#} \mathrm{P}<0.01$. ASMq, Abnormal Savda Munziq; Cyto C, cytochrome c; p-, phosphorylated; ns, not significant.

Erk/p90 ribosomal S6 kinase (p90RSK) pathway acts upstream in the regulation of $A S M q$ on mitochondria-associated apoptosis in the zone of stasis. As indicated by immunofluorescence staining, the distribution of cells positive for p-Erk was demonstrated by an elevation in green-stained cells in the interspace skin following burn injury (Fig. 5). All three dosages of ASMq notably enhanced the phosphorylation of Erk, and the highest dose $(1,600 \mathrm{mg} / \mathrm{kg})$ exerted the strongest effect (Fig. 5).

The results of western blotting were consistent with immunofluorescence staining: ASMq dose-dependently increased protein expression levels of p-Erk and p90RSK. PD98059 reversed the elevated expression of p-Erk and p90RSK induced by ASMq (Fig. 6).

\section{Discussion}

Uygur medicine, one of the most important branches of Chinese traditional medicine, provides ways to manage emergency situations and disease (16,30-32). In the systemic theory of Uyghur medicine, the liver produces bodily fluids with different effects that can be divided into four types: Sapra, kan hiliti, phlegm and sawda $(15,16)$. There is a complementary balance between these fluids, which serves a role in the maintenance of normal physiology and the occurrence and development of disease. When the internal environment changes, these four body fluids become AS, which can be harmful to various organs (10). With advancements of science and technology, research into Uyghur traditional medicine has become more in-depth, with significant progress in drug extraction and component analysis (33-35). ASMq, a natural compound of traditional Uyghur Medicine, has been reported to exhibit anti-inflammatory, antioxidant and anti-apoptotic effects, and decreases oxidative damage by free radicals (10-17). The production of ASMq is subject to a patent application (no. ZL02130082.8) in China (10).

Deep burn injury tends to induce the formation of scars, especially hypertrophic scars, which are related to damage of the dermis $(3,6)$. Early intervention before progressive changes of initial deep burn wounds can prevent further deepening and extension, decrease damage of the dermis and subcutaneous tissue and prevent the generation of secondary hypertrophic scars (36). Based on previously reported anti-inflammatory, anti-apoptotic and antioxidant effects of ASMq in the treatment of various diseases $(4,15,23,37)$ and the underlying mechanism

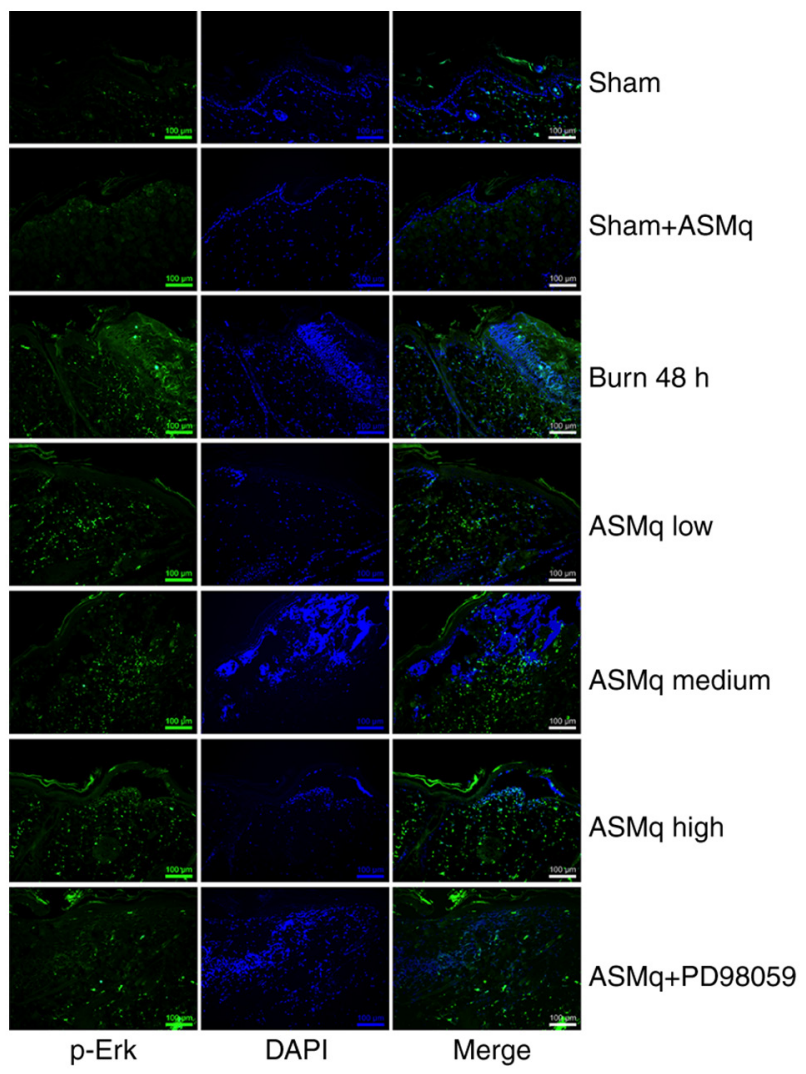

Figure 5. Effect of ASMq on the distribution and expression of p-Erk in the zone of stasis. Representative images of immunofluorescence staining of p-Erk. Scale bar, $100 \mu \mathrm{m}$. ASMq, Abnormal Savda Munziq; p-, phosphorylated.

of early burn wound progression, it was hypothesized that ASMq may have a potential therapeutic effect on the progressive deepening of early burn wounds. Oxygen free radicals are considered to be important mediators of tissue damage progression following thermal injury $(9,38)$. In addition to thermal injury, XO and Nox participate in the regulation of oxygen free radicals (9). ROS induce cell death in the zone of stasis via lipid peroxidation and protein degradation (8). Moreover, ROS have been reported to disrupt the mitochondrial outer membrane, leading to the release of Cyto C and the activation of apoptosis $(39,40)$. The current focus of research is the administration of antioxidants to alleviate oxidative stress of burn wounds, and thus prevent the progress 

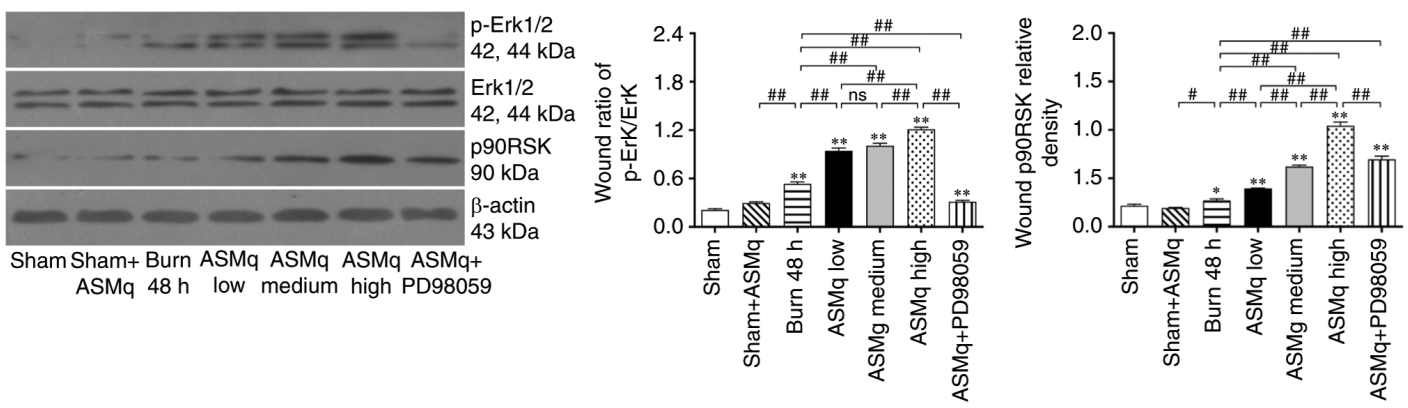

Figure 6. PD98059 reverses ASMq-mediated upregulation of Erk phosphorylation and p90RSK expression in the zone of stasis post-burn. Western blot bands and relative $\mathrm{p}$-Erk/Erk ratios and p90RSK levels. Data are presented as the mean $\pm \mathrm{SD}$ (n=8/group). ${ }^{*} \mathrm{P}<0.05$ and ${ }^{* *} \mathrm{P}<0.01$ vs. sham; ${ }^{*} \mathrm{P}<0.05$, ${ }^{\# \#} \mathrm{P}<0.01$. ASMq, Abnormal Savda Munziq; p-, phosphorylated; ns, not significant; p90RSK, P90 ribosomal S6 kinase.

of wound deterioration $(34,41)$. Guo et al (9) demonstrated that hydrogen-rich saline, a specific free radical scavenger, decreases lipid peroxidation and enhances the activity of endogenous antioxidant enzymes, thus alleviating early wound progression following deep burn injury in vivo. Previous reports demonstrated that ASMq attenuates mitochondrial damage caused by free radicals $(12,13)$. Certain ingredients (such as tannins, vitamins and flavones) in ASMq prevent free radical chain reaction, scavenge free radicals and decrease the production of -OH (11). Ischemia/reperfusion injury elevates levels of oxidative stress in tissue and induces cell damage, such as lipid peroxidation (37,42). Abudunaibi et al (13) reported that ASMq effectively ameliorates lipid peroxidation and oxidative stress in rats with myocardial ischemia/reperfusion injury. ASMq also shows significant dose-dependent antioxidative effects (37). In the present study, it was observed that both medium and high dosage ASMq attenuated lipid peroxidation of skin tissue in the zone of stasis, and the high dosage group showed more significant effects. Although low and medium dosage ASMq showed limited effects on increasing burn-induced decreases in endogenous antioxidative enzyme activity, high dosage ASMq significantly enhanced the activity of endogenous antioxidant enzymes (GPx and SOD) in the zone of stasis of rat burn wounds. Additionally, by investigating potential in vivo effects of ASMq on the generation pathway of oxidative free radicals $\mathrm{XO}$ and Nox, it was found that ASMq had a notable effect on decreasing oxidative free radicals by dose-dependently downregulating the expression levels of $\mathrm{XO}$ and Nox.

Apoptosis serves a key role in early burn wound progression secondary to burn-induced oxidative stress (43). Before irreversible cell inactivation occurs, appropriate drug intervention can alleviate cell apoptosis in the zone of stasis $(9,41)$. Studies have attempted to ameliorate the progressive deepening of burn wounds by inhibiting apoptosis $(6,41)$. Our previous study (9) illustrated that hydrogen-rich saline exerted a potent effect on ameliorating apoptosis in the zone of stasis and prevented wound deepening and extension. In the present study, TUNEL staining was performed to investigate the distribution of apoptotic cells in the zone of stasis directly and evaluate the effect of ASMq on the number of apoptotic cells in the burn wound. The results indicated that ASMq dose-dependently decreased cell apoptosis in the burn wound tissue of rats. The caspase family are important signaling molecules of the cell apoptotic pathway (29). Immunofluorescence staining demonstrated that medium and high dosage ASMq decreased the distribution

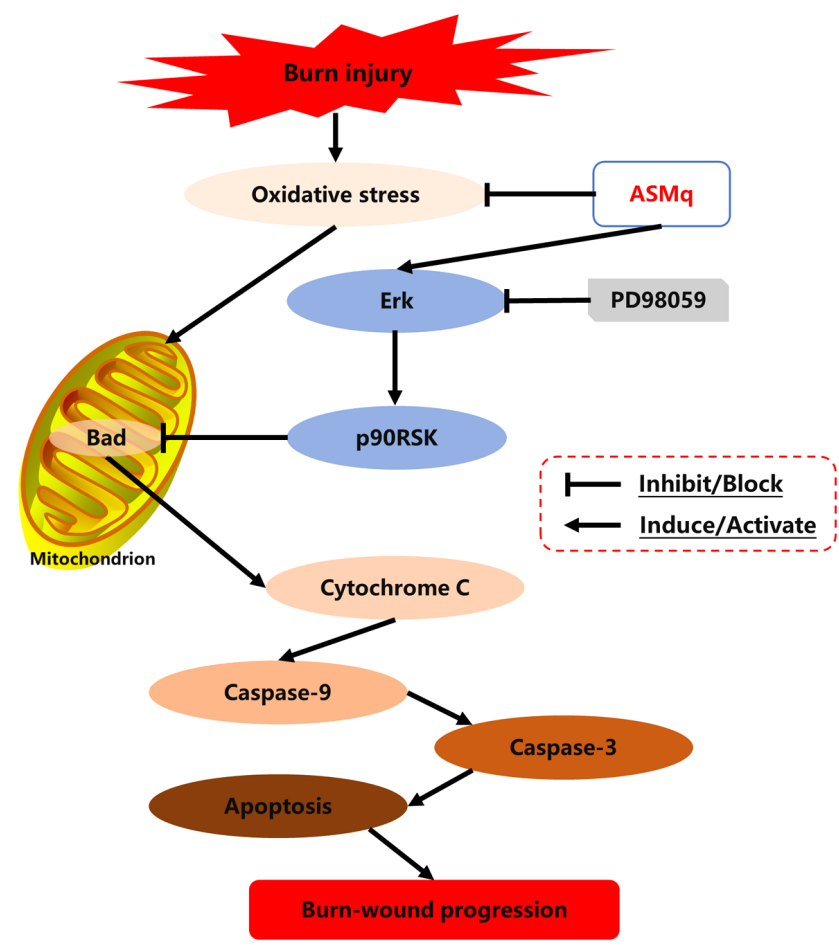

Figure 7. Schematic diagram of the potential mechanisms of burn-wound progression and the routes by which ASMq exerts its effects. ASMq, Abnormal Savda Munziq; p90RSK, P90 ribosomal S6 kinase.

of $\mathrm{CC} 3$ significantly. In agreement with the aforementioned results, western blotting showed that ASMq dose-dependently reversed the increased expression of CC 3 and CC9 in burn wounds. These results supported the protective effect of ASMq on tissue apoptosis in the zone of stasis. In addition, the release of Cyto $\mathrm{C}$ was also detected in the burn wound. As a proapoptotic protein, Cyto $\mathrm{C}$ is released by mitochondria under cellular stress and combines with apoptotic peptidase activating factor 1 and caspase- 9 to form an activated complex, which cleaves caspase- 3 into $\mathrm{CC} 3$ (an activated state) and induces apoptosis (29). Here, ASMq exhibited the ability to lower cytosolic levels of Cyto $\mathrm{C}$ at all dosages.

As a member of the proapoptotic BH3-only subfamily, $\mathrm{Bad}$ is involved in the induction of apoptotic cascades $(44,45)$. Dephosphorylated Bad binds to anti-apoptotic Bcl-2 and $\mathrm{Bcl}-\mathrm{xL}$ proteins, then inactivates these proteins and induces pro-apoptotic Bax protein translocation from the cytosol to 
the mitochondria, which results in the release of Cyto $\mathrm{C}$ from mitochondria into the cytoplasm, and subsequently activates the caspase cascade $(42,46)$. p-Bad can inhibit the combination of Bad with anti-apoptotic Bcl-2 and Bcl-xL proteins at the mitochondrial outer membrane, thereby blocking the Bax-induced Cyto C/caspase-mediated apoptotic pathway $(46,47)$. In the current study, ASMq increased the ratio of $\mathrm{p}-\mathrm{Bad} / \mathrm{Bad}$, which indicated that Bad dissociated from Bcl-2 and $\mathrm{Bcl}-\mathrm{xL}$ via phosphorylation of the Serine-136 residue of $\operatorname{Bad}(48,49)$, thus downregulating the expression levels of Cyto C. In all, the mitochondria-associated apoptotic pathway seemed to be activated in the process of burn wound conversion. MAPK, a type of serine/threonine protein kinase in eukaryotes, serves a vital role in transducing extracellular stimuli into intracellular signals, which cause various biochemical reactions, such as proliferation, differentiation and apoptosis in cells $(45,50)$. Erk is an important member of the MAPK signaling pathway (45). p90RSK, a downstream target of MAPK that is phosphorylated and activated by $\mathrm{p}$-Erk1/2, has been reported to be involved in the phosphorylation of Bad and subsequent inactivation of the pro-apoptotic function of $\mathrm{Bad}(42,46,47)$. The present study found that the phosphorylation of Erk and expression of p90RSK was increased in the zone of stasis following burn injury, which indicated that the Erk/p90RSK signaling pathway may be an endogenous defense mechanism against apoptosis caused by thermal injury. Furthermore, the Erk inhibitor PD98059 notably reversed the beneficial effect of ASMq on cell apoptosis and expression levels of $\mathrm{CC} 3$ and $\mathrm{CC} 9$ in the zone of stasis following burn injury. Meanwhile, the effect of PD98059 was associated with its inhibition of Erk phosphorylation and p90RSK expression levels and promotion of Bad-induced Cyto $\mathrm{C}$ release. These results suggested that the Erk/p90RSK/Bad signaling pathway may play a crucial role in the underlying mechanism of ASMq in the regulation of mitochondria-associated apoptosis. A schematic diagram of the potential mechanisms uncovered by the present study is presented in Fig. 7.

However, there are a number of limitations of the present study: Quantitative analysis of histological changes were not performed, ultrastructural changes of mitochondria and the metabolites of ASMq in vivo were not detected, expression levels of ROS, nitric oxide (NO) and inducible NO synthase were not detected directly and changes in other potential signaling molecules were not assessed. Further investigation should be considered to obtain in-depth details of the underlying mechanism. Overall, ASMq administration contributed to the attenuation of burn wound progression following thermal injury in a dose-dependent manner. The protective mechanism was shown to be associated with the antioxidative and anti-apoptotic effect of ASMq and the regulation of the mitochondria-associated apoptotic pathway by ASMq, with the involvement of upstream Erk/p90RSK activation.

\section{Acknowledgements}

Not applicable.

\section{Funding}

The present study was supported by the National Natural Science Foundation of China (grant nos. 81671909 and 81901958),
Zhejiang Provincial Natural Science Foundation of China (grant nos. LY18H150004, LY19H150004 and LY20H150010) and Key Research and Development Project of Sichuan Science and Technology Department (grant no. 2018SZ0380).

\section{Availability of data and materials}

The analyzed datasets generated during the study are available from the corresponding author on reasonable request.

\section{Authors' contributions}

HZ, QF and SG conceptualized and designed the present study and provided administrative support. HZ, NL, HC and MY made contributions to the acquisition of data. $\mathrm{HZ}$ and SG analyzed and interpreted the data. HZ, QF and NL were involved in drafting the manuscript. HC, MY and SG revised the manuscript critically for important intellectual content. QF and SG confirm the authenticity of all the raw data. All authors read and approved the final manuscript.

\section{Ethics approval and consent to participate}

The present study was protocol approved by The Second Affiliated Hospital, Zhejiang University, School of Medicine, Institutional Animal Care and Use Committee (approval no. 2018-095) in accordance with the National Institutes of Health Guidelines for the Care and Use of Laboratory Animals.

\section{Patient consent for publication}

Not applicable.

\section{Competing interests}

The authors declare that they have no competing interests.

\section{References}

1. Jackson DM: The diagnosis of the depth of burning. Br J Surg 40: 588-596, 1953 (In Undetermined language)

2. Singh V, Devgan L, Bhat S and Milner SM: The pathogenesis of burn wound conversion. Ann Plast Surg 59: 109-115, 2007.

3. Johnson RM and Richard R: Partial-thickness burns: Identification and management. Adv Skin Wound Care 16: 178-189, 2003.

4. Li N, Kong M, Ma T, Gao W and Ma S: Uighur medicine abnormal savda munzip (ASMq) suppresses expression of collagen and TGF- $\beta 1$ with concomitant induce Smad7 in human hypertrophic scar fibroblasts. Int J Clin Exp Med 8: 8551-8560, 2015.

5. Singer AJ, McClain SA, Taira BR, Guerriero JL and Zong W: Apoptosis and necrosis in the ischemic zone adjacent to third degree burns. Acad Emerg Med 15: 549-554, 2008.

6. Arturson G: Pathophysiology of the burn wound and pharmacological treatment. The Rudi hermans lecture, 1995. Burns 22: 255-274, 1996.

7. Bhatti JS, Bhatti GK and Reddy PH: Mitochondrial dysfunction and oxidative stress in metabolic disorders-A step towards mitochondria based therapeutic strategies. Biochim Biophys Acta Mol Basis Dis 1863: 1066-1077, 2017.

8. Fang Q, Guo S, Zhou H, Han R, Wu P and Han C: Astaxanthin protects against early burn-wound progression in rats by attenuating oxidative stress-induced inflammation and mitochondria-related apoptosis. Sci Rep 7: 41440, 2017.

9. Guo SX, Jin YY, Fang Q, You CG, Wang XG, Hu XL and Han CM: Beneficial effects of hydrogen-rich saline on early burn-wound progression in rats. PLoS One 10: e0124897, 2015. 
10. Hao Y, Xiao Y, Zhang Z, Zhao H, Zhou W, Guo X, Lu A and $\mathrm{Li}$ X: Transition of the abnormal savda syndrome to the hepatic carcinoma shifted unfolded protein response to autophagy was partly reversed by savda munziq in a rat model. Biomed Pharmacother 121: 109643, 2020.

11. Yusup A, Upur H, Umar A and Moore N: Protective effects of munziq and mushil of abnormal savda to mitochondrial oxidative damage. Fundam Clin Pharmacol 18: 471-476, 2004.

12. Abliz G, Mijit F, Hua L, Abdixkur G, Ablimit T, Amat N and Upur H: Anti-carcinogenic effects of the phenolic-rich extract from abnormal savda munziq in association with its cytotoxicity, apoptosis-inducing properties and telomerase activity in human cervical cancer cells (SiHa). BMC Complement Altern Med 15: 23, 2015.

13. Abudunaibi M, Mulati A, Aisikaer S, Zulifeiya M, Qiao J, Gulibositan M, Aili A and Halmurat U: Myocardial protective effects of munziq in myocardial ischemia-reperfusion injury rats with abnormal savda syndrome. Genet Mol Res 14: 3426-3435, 2015.

14. Aikemu A, Amat N, Yusup A, Shan L, Qi X and Upur H: Attenuation effect of abnormal savda munziq on liver and heart toxicity caused by chemotherapy in mice. Exp Ther Med 12: 384-390, 2016.

15. Omarniyaz Z, Yu Y, Yang T, Shan L, Miao W, Reyimu R, Upur H and Aikemu A: Anti-tumor effects of abnormal savda munzic on the transplanted cervical cancer (U27) mouse model. BMC Complement Altern Med 16: 477, 2016.

16. Shabiti A, Aibibula A, Tuerxun A and Wufuer H: Therapeutic effect and mechanism of action of abnormal savda munziq in development of degenerative atherosclerotic aortic valve disease. Med Sci Monit 23: 4431-4439, 2017.

17. Maimaitiaili A, Li J, Aibibula A and Abudureheman M Inhibition of nuclear factor kappa B pathway protects myocardial ischemia/reperfusion injury in rats under treatment with abnormal savda munziq. Am J Transl Res 10: 77-85, 2018.

18. National Institutes of Health: Guide for the care and use of laboratory animals. 8th edition, Institute for Laboratory Animal Research, Division on Earth and Life Studies, National Research Council of the National Academies Press, Washington, DC, pp1-246, 2011

19. Eski M, Ozer F, Firat C, Alhan D, Arslan N, Senturk T and Işik S: Cerium nitrate treatment prevents progressive tissue necrosis in the zone of stasis following burn. Burns 38: 283-289, 2012.

20. Singer AJ, Towery H and McClain SA: Effect of tadalafil on reduction of necrosis in the ischemic zone in a rat comb burn model. Burns 44: 1427-1432, 2018.

21. Reddy AS, Abraham A, McClain SA, Clark RA, Ralen P, Sandoval S and Singer AJ: The role of necroptosis in burn injury progression in a rat comb burn model. Acad Emerg Med 22: 1181-1186, 2015

22. Regas FC and Ehrlich HP: Elucidating the vascular response to burns with a new rat model. J Trauma 32: 557-563, 1992.

23. Yusup A, Upur H, Umar A, Berke B, Yimit D, Lapham JC Moore N and Cassand P: Abnormal savda munziq, an herbal preparation of traditional uighur medicine, may prevent 1,2-dimethylhydrazine-induced rat colon carcinogenesis. Evid Based Complement Alternat Med 2011: 152015, 2011.

24. Hou L, Hou X, Wang L, Li Z, Xin B, Chen J, Gao X and Mu H: PD98059 impairs the cisplatin-resistance of ovarian cancer cells by suppressing ERK pathway and epithelial mesenchymal transition process. Cancer Biomark 21: 187-194, 2017.

25. Close B, Banister K, Baumans V, Bernoth EM, Bromage N, Bunyan J, Erhardt W, Flecknell P, Gregory N, Hackbarth H, et al: Recommendations for euthanasia of experimental animals: Part 1. DGXI of the European commission. Lab Anim 30: 293-316, 1996.

26. Tsikas D: Assessment of lipid peroxidation by measuring malondialdehyde (MDA) and relatives in biological samples: Analytical and biological challenges. Anal Biochem 524: 13-30, 2017.

27. Retamoso LT, Silveira Junior MEP, Lima FD, Busanello GL, Bresciani G, Ribeiro LR, Chagas PM, Nogueira CW, Braga AC, Furian AF, et al: Increased xanthine oxidase-related ROS production and TRPV1 synthesis preceding DOMS post-eccentric exercise in rats. Life Sci 152: 52-59, 2016

28. Sun J, Chen J, Li T, Huang P, Li J, Shen M, Gao M, Sun Y, Liang J, Li X, et al: ROS production and mitochondrial dysfunction driven by PU.1-regulated NOX4-p22 $2^{\text {phox }}$ activation in A $\beta$-induced retinal pigment epithelial cell injury. Theranostics 10: 11637-11655, 2020

29. Hengartner MO: The biochemistry of apoptosis. Nature 407: 770-776, 2000

30. Ma ZQ, Hu H, He TT, Guo H, Zhang MY, Chen MW and Wang YT: An assessment of traditional uighur medicine in current Xinjiang region (China). Afr J Tradit Complement Altern Med 11: 301-314, 2014.
31. Mamtimin B, Xia G, Mijit M, Hizbulla M, Kurbantay N, You L and Upur H: Metabolic differentiation and classification of abnormal savda munziq's pharmacodynamic role on rat models with different diseases by nuclear magnetic resonance-based metabonomics. Pharmacogn Mag 11: 698-706, 2015.

32. Zhen G, Upur H, Jing W, Jing J, Zheng L, Dan X and Fengsen L: Effect of abnormal savda munziq, a traditional uighur herbal medicine, on pulmonary function and aquaporins of COPD rat model with abnormal savda syndrome. Evid Based Complement Alternat Med 2017: 7176263, 2017.

33. Chaudhary A, Wang J and Prabhu S: Development and validation of a high-performance liquid chromatography method for the simultaneous determination of aspirin and folic acid from nano-particulate systems. Biomed Chromatogr 24: 919-925, 2010.

34. Kizaibek M, Popescu R, Prinz S, Upur H, Singhuber J, Zehl M and Kopp B: Towards modernization of the formulation of the traditional uighur medicine herbal preparation abnormal savda munziq. Evid Based Complement Alternat Med 2012: 863101, 2012.

35. Guo X, Bakri I, Abudula A, Arken K, Mijit M, Mamtimin B and Upur H: Differential integrative omic analysis for mechanism insights and biomarker discovery of abnormal savda syndrome and its unique munziq prescription. Sci Rep 6: 27831, 2016.

36. Shupp JW, Nasabzadeh TJ, Rosenthal DS, Jordan MH, Fidler P and Jeng JC: A review of the local pathophysiologic bases of burn wound progression. J Burn Care Res 31: 849-873, 2010.

37. Maimaitiaili A, Shabiti A, Abudureheman M, Musha Z, Jun Q, Maimaitiaili G, Aibibula A and Upur H: Effects of different doses of savda munziq on myocardial ischemia-reperfusion injury in rats with abnormal savda syndrome. Genet Mol Res 13: 4729-4735, 2014

38. Guo SX, Fang Q, You CG, Jin YY, Wang XG, Hu XL and Han CM: Effects of hydrogen-rich saline on early acute kidney injury in severely burned rats by suppressing oxidative stress induced apoptosis and inflammation. J Transl Med 13: 183, 2015

39. Lei X, Chao H, Zhang Z, Lv J, Li S, Wei H, Xue R, Li F and Li Z: Neuroprotective effects of quercetin in a mouse model of brain ischemic/reperfusion injury via anti-apoptotic mechanisms based on the Akt pathway. Mol Med Rep 12: 3688-3696, 2015.

40. Arabian M, Aboutaleb N, Soleimani M, Mehrjerdi FZ, Ajami M and Pazoki-Toroudi $\mathrm{H}$ : Role of morphine preconditioning and nitric oxide following brain ischemia reperfusion injury in mice. Iran J Basic Med Sci 18: 14-21, 2015.

41. Salibian AA, Rosario ATD, Severo LAM, Nguyen L, Banyard DA, Toranto JD, Evans GRD and Widgerow AD: Current concepts on burn wound conversion-A review of recent advances in understanding the secondary progressions of burns. Burns 42 : 1025-1035, 2016.

42. Cheng CY, Kao ST and Lee YC: Angelica sinensis extract protects against ischemia-reperfusion injury in the hippocampus by activating p38 MAPK-mediated p90RSK/p-Bad and p90RSK/CREB/BDNF signaling after transient global cerebral ischemia in rats. J Ethnopharmacol 252: 112612, 2020.

43. Tan JQ, Zhang HH, Lei ZJ, Ren P, Deng C, Li XY and Chen SZ: The roles of autophagy and apoptosis in burn wound progression in rats. Burns 39: 1551-1556, 2013.

44. Koh PO: Melatonin attenuates the cerebral ischemic injury via the MEK/ERK/p90RSK/bad signaling cascade. J Vet Med Sci 70: 1219-1223, 2008.

45. Raman M, Chen W and Cobb MH: Differential regulation and properties of MAPKs. Oncogene 26: 3100-3112, 2007.

46. Koh PO: Ferulic acid attenuates the down-regulation of MEK/ERK/p90RSK signaling pathway in focal cerebral ischemic injury. Neurosci Lett 588: 18-23, 2015.

47. YuZ,CaiM,LiX,ZhangJ,WuT, Yang F,ZhuW,Xiang Y,Zhang W, Xiang $\mathbf{J}$ and Cai D: Neuroprotective effects of Tongxinluo on focal cerebral ischemia and reperfusion injury in rats associated with the activation of the MEK1/2/ERK1/2/p90RSK signaling pathway. Brain Res 1685: 9-18, 2018.

48. Gross A: BCL-2 family proteins as regulators of mitochondria metabolism. Biochim Biophys Acta 1857: 1243-1246, 2016.

49. Chalah A and Khosravi-Far R: The mitochondrial death pathway. Adv Exp Med Biol 615: 25-45, 2008.

50. Alexander M, Daniel T, Chaudry IH and Schwacha MG: MAP kinases differentially regulate the expression of macrophage hyperactivity after thermal injury. J Cell Physiol 201: 35-44, 2004. 